\title{
Premature Atrial Contractions on the Screening Electrocardiogram and Risk of Ischemic Stroke: The Reasons for Geographic and Racial Differences in Stroke Study
}

\author{
Wesley T. O'Neal ${ }^{\mathrm{a}}$ Hooman Kamel ${ }^{\mathrm{b}}$ Dawn Kleindorfer ${ }^{\mathrm{c}}$ Suzanne E. Judd ${ }^{\mathrm{d}}$ \\ George Howard $^{d}$ Virginia J. Howard $^{e}$ Elsayed Z. Soliman ${ }^{f}$ \\ a Department of Medicine, Division of Cardiology, Emory University School of Medicine, Atlanta, Ga., ${ }^{\text {b Department }}$ \\ of Neurology, Weil Cornell Medical College, New York, N.Y., 'Department of Neurology, University of Cincinnati, \\ Cincinnati, Ohio, dDepartment of Biostatistics, School of Public Health, University of Alabama at Birmingham, and \\ e Department of Epidemiology, School of Public Health, University of Alabama at Birmingham, Birmingham, Ala., \\ and ${ }^{f}$ Epidemiological Cardiology Research Center (EPICARE), Department of Epidemiology and Prevention, and \\ Department of Internal Medicine, Section on Cardiology, Wake Forest School of Medicine, Winston-Salem, N.C., USA
}

\section{Key Words}

Atrial ectopy $\cdot$ Stroke $\cdot$ Epidemiology

\begin{abstract}
Background: It is currently unknown if premature atrial contractions (PACs) detected on the routine screening electrocardiogram are associated with an increased risk of ischemic stroke. Methods: We examined the association between PACs and ischemic stroke in 22,975 (mean age $64 \pm 9.2 ; 56 \%$ women; $40 \%$ black) participants from the Reasons for Geographic and Racial Differences in Stroke study. Participants who were free of stroke at baseline were included. PACs were detected from centrally read electrocardiograms at baseline. Cox regression was used to examine the association between PACs and ischemic stroke events through March 31, 2014. Results: PACs were present in 1,687 (7.3\%) participants at baseline. In a Cox regression model adjusted for stroke risk factors and potential confounders, PACs were associated with an increased risk of ischemic stroke (hazards ratio (HR) $1.34,95 \% \mathrm{Cl} 1.04-1.74)$. The relationship was lim-
\end{abstract}

ited to non-lacunar infarcts ( $\mathrm{HR} 1.42,95 \% \mathrm{Cl} 1.08-1.87)$, and not lacunar strokes (HR 1.01, 95\% Cl 0.51-2.03). An interaction by sex was detected, with the association between PACs and ischemic stroke being stronger among women (HR 1.82, 95\% Cl 1.29-2.56) than men (HR 1.03, 95\% Cl 0.69-1.52; pinteraction $=0.0095)$. Conclusion: $P A C s$ detected on the routine electrocardiogram are associated with an increased risk for non-lacunar ischemic strokes, especially in women.

๑) 2016 S. Karger AG, Basel

\section{Introduction}

Premature atrial contractions (PACs) are frequently found on the electrocardiograms of patients with ischemic stroke, suggesting a potential link between atrial ectopy and stroke [1]. Despite the well-known association between PACs and atrial fibrillation [2-4], the stroke risk associated with PACs remains after accounting for incident atrial fibrillation [5]. This suggests that atrial ectopic beats possibly are independently associated with cardio-

\section{KARGER}

E-Mail karger@karger.com

www.karger.com/ned
C 2016 S. Karger AG, Basel

$0251-5350 / 16 / 0471-0053 \$ 39.50 / 0$
Wesley T. O'Neal, MD

Department of Medicine, Division of Cardiology Emory University School of Medicine, 101 Woodruff Circle Woodruff Memorial Building, Atlanta, GA 30322 (USA)

E-Mail wesley.oneal@emory.edu 
embolic stroke, and provides further support that left atrial disease is associated with thromboembolic complications (e.g., atrial cardiopathy). This is further supported by prior studies showing that frequent atrial ectopy detected by 48 -hour ambulatory electrocardiography is associated with an increased risk of ischemic stroke $[5,6]$.

It is currently unknown if PACs detected on a routine screening electrocardiogram are associated with an increased risk of ischemic stroke. Such a finding would have important implications regarding stroke risk for persons with atrial ectopy detected on the resting electrocardiogram, as long-term electrocardiographic monitoring is not possible in many patient populations. Therefore, the purpose of this study was to examine the association between PACs and ischemic stroke in the Reasons for Geographic and Racial Differences in Stroke (REGARDS) study, one of the largest biracial, prospective cohort studies in the United States.

\section{Methods}

\section{Study Population and Design}

Details of REGARDS have been published previously [7]. Briefly, REGARDS was designed to identify causes of regional and racial disparities in stroke mortality. Between January 2003 and October 2007, the study recruited 30,239 participants, with an over sampling of blacks and residents of the stroke belt (North Carolina, South Carolina, Georgia, Alabama, Mississippi, Tennessee, Arkansas and Louisiana). Potential participants were recruited from a commercially available list of residents using postal mailings and telephone contact. Demographic information and medical history were obtained using a computer-assisted telephone interview system that was conducted by trained interviewers. Additionally, a brief inhome physical examination was performed 3-4 weeks after the telephone interview. During the in-home visit, trained staff obtained information on medications taken within the past 2 weeks. Also, blood and urine samples, a resting electrocardiogram, and anthropometric measurements were collected. For the purpose of this analysis, participants were excluded for the following reasons: data inconsistencies $(n=56)$, prevalent baseline stroke/transient ischemic attack $(n=3,044)$, missing follow-up data $(n=438)$, atrioventricular blocks and evidence of non-sinus rhythm on the baseline electrocardiogram (e.g., atrial fibrillation, atrial flutter, wandering atrial pacemaker; $\mathrm{n}=597)$, or missing baseline characteristics $(\mathrm{n}=$ 1,690). Additionally, participants with frequent premature ventricular contractions $(n=1,439)$ were excluded due to the reported association between ventricular ectopy and ischemic stroke [8].

\section{Premature Atrial Contractions}

PACs were detected on the baseline electrocardiogram and defined by Minnesota code criteria (Minnesota code 8.1.1) [9]. PACs were classified as present or absent. All electrocardiograms were centrally read at the Epidemiological Cardiology Research Center (Wake Forest School of Medicine, Winston-Salem, N.C., USA) by readers who were blinded to study outcomes.

\section{Ischemic Stroke Events}

The adjudication process for stroke events in REGARDS has been previously reported [10]. Briefly, during follow-up, reports of possible strokes, transient ischemic attacks, deaths, hospitalization or emergency department visits for stroke symptoms, or hospitalization for unknown reasons generated a request for medical record review. Possible stroke events were centrally adjudicated by a team of physicians. For deaths without medical records, death certificates and/or proxy interviews were used to adjudicate events. Strokes were defined using the World Health Organization (WHO) definition [11]. Events that did not meet the WHO definition, but with symptoms lasting more than $24 \mathrm{~h}$ and with imaging consistent with acute ischemia or hemorrhage, were classified as 'clinical strokes'. This analysis included WHO and clinical ischemic strokes. All ischemic strokes were further classified as lacunar or non-lacunar.

\section{Covariates}

Age, sex, race and smoking status were self-reported. Smoking was defined as current tobacco use. Fasting blood samples were obtained and assayed for low-density lipoprotein and serum glucose. Diabetes was defined as a fasting glucose level $\geq 126 \mathrm{mg} / \mathrm{dl}$ (or a non-fasting glucose $\geq 200 \mathrm{mg} / \mathrm{dl}$ among those failing to fast) or self-reported diabetes medication use. The current use of aspirin, antihypertensive medications, and lipid-lowering therapies was self-reported. After the participant rested for $5 \mathrm{~min}$ in a seated position, blood pressure was measured using a sphygmomanometer. Two values were obtained following a standardized protocol and averaged. Body mass index was defined as the weight in kilograms divided by the height in meters squared. Coronary heart disease was defined by self-reported history of myocardial infarction, coronary artery bypass grafting, coronary angioplasty or stenting, or evidence of prior myocardial infarction on the baseline electrocardiogram.

\section{Statistical Analyses}

Baseline characteristics were compared by the presence of PACs. Categorical variables were reported as frequency and percentage while continuous variables were reported as mean \pm SD. Statistical significance for categorical variables was tested using the chi-square method and the Student's t test for continuous variables. Incidence rates per 1,000 person-years were computed for ischemic stroke by the presence of PACs. Kaplan-Meier estimates were used to compute the cumulative incidence of ischemic stroke for each category and the differences in estimates were compared using the log-rank procedure. Follow-up time was defined as the time between the baseline electrocardiogram measurement until a diagnosis of ischemic stroke, death, loss to follow-up or end of follow-up (March 31, 2014). Cox regression was used to compute hazards ratios (HRs) and 95\% CIs for the association between PACs and ischemic stroke. Multivariable models were adjusted as follows: Model 1 adjusted for age, sex, race, age*race, education, income and region of residence; and Model 2 adjusted for Model 1 covariates plus systolic blood pressure, smoking, diabetes, low-density lipoprotein cholesterol, left ventricular hypertrophy, antihypertensive medications, lipid-lowering therapies, aspirin, and coronary heart disease. We also examined the association between PACs and ischemic stroke by stroke subtype (e.g., lacunar and non-lacunar) since cardiac embolism typically results in large or cortical 
Table 1. Baseline characteristics PACs $(n=22,975)$

\begin{tabular}{|c|c|c|c|}
\hline Baseline characteristic & $\begin{array}{l}\text { PACs } \\
(n=1,687)\end{array}$ & $\begin{array}{l}\text { No PACs } \\
(n=21,288)\end{array}$ & $\mathrm{p}$ value \\
\hline Age, years, mean $\pm S D$ & $69 \pm 9.6$ & $64 \pm 9.1$ & $<0.0001$ \\
\hline Male, n (\%) & $844(50)$ & $9,206(43)$ & $<0.0001$ \\
\hline Black, n (\%) & $727(43)$ & $8,539(40)$ & 0.016 \\
\hline Region & & & 0.017 \\
\hline Stroke buckle, n (\%) & $354(21)$ & $4,497(21)$ & \\
\hline Stroke belt, $\mathrm{n}(\%)$ & $541(32)$ & $7,494(35)$ & \\
\hline Non-belt, $\mathrm{n}(\%)$ & $792(47)$ & $9,297(44)$ & \\
\hline Education, high school or less, n (\%) & $673(40)$ & $7,730(36)$ & \\
\hline Income, $<\$ 20,000, \mathrm{n}(\%)$ & $308(18)$ & $3,427(16)$ & 0.021 \\
\hline Ever smoker, n (\%) & $920(55)$ & $11,340(53)$ & 0.32 \\
\hline Diabetes, $\mathrm{n}(\%)$ & $379(22)$ & $3,980(19)$ & 0.0001 \\
\hline Systolic blood pressure, $\mathrm{mm} \mathrm{Hg}$, mean $\pm \mathrm{SD}$ & $130 \pm 18$ & $127 \pm 16$ & $<0.0001$ \\
\hline Body mass index, $\mathrm{kg} / \mathrm{m}^{2}$, mean $\pm \mathrm{SD}$ & $29 \pm 6.3$ & $29 \pm 6.1$ & 0.025 \\
\hline Low-density lipoprotein, $\mathrm{mg} / \mathrm{dl}$, mean $\pm \mathrm{SD}$ & $112 \pm 34$ & $115 \pm 35$ & 0.0012 \\
\hline Aspirin, $\mathrm{n}(\%)$ & $753(45)$ & $8,644(41)$ & 0.0012 \\
\hline Lipid-lowering therapies, n (\%) & $543(32)$ & $6,642(31)$ & 0.40 \\
\hline Antihypertensive medications, $\mathrm{n}(\%)$ & $908(54)$ & $10,171(48)$ & $<0.0001$ \\
\hline Left ventricular hypertrophy, n (\%) & $179(11)$ & $1,916(9.0)$ & 0.027 \\
\hline Coronary heart disease, $\mathrm{n}(\%)$ & $320(19)$ & $3,013(14)$ & $<0.0001$ \\
\hline
\end{tabular}

Statistical significance for categorical variables tested using the chi-square method, and for continuous variables, the Student's t test was used.

Table 2. Risk of ischemic stroke associated with PACs $(\mathrm{n}=22,975)$

\begin{tabular}{lrllllll}
\hline PAC & Cases & Person-years & $\begin{array}{l}\text { Incidence rate, per } \\
1,000 \text { person-years }\end{array}$ & $\begin{array}{l}\text { Model } 1^{*}, \\
\text { HR }(95 \% \mathrm{CI})\end{array}$ & p value & $\begin{array}{l}\text { Model 2 } \\
\text { HR }(95 \% \text { CI })\end{array}$ & p value \\
\hline Absent & 481 & 152,489 & $3.2(2.9-3.4)$ & 1.0 & - & 1.0 & - \\
Present & 68 & 11,299 & $6.0(4.7-7.6)$ & $1.37(1.06-1.77)$ & 0.018 & $1.34(1.04-1.74)$ & 0.025 \\
\hline
\end{tabular}

* Adjusted for age, sex, race, age*race, education, income and region of residence.

$\dagger$ Adjusted for Model 1 covariates plus systolic blood pressure, smoking, diabetes, low-density lipoprotein cholesterol, left ventricular hypertrophy, antihypertensive medications, lipid-lowering therapies, aspirin, and coronary heart disease.

strokes rather than small subcortical strokes [12]. Additionally, we evaluated effect modification by age, sex, and race, using a stratification technique and comparing models with and without interaction terms. Due to the association between premature ventricular contractions and ischemic stroke [8], a sensitivity analysis was performed with further adjustment for concomitant ventricular ectopy. We also examined the relationship between PACs and recurrent ischemic stroke in those who had a self-reported history of stroke at baseline $(n=1,468)$. The proportional hazards assumption was not violated in our analysis. Statistical significance for all comparisons including interactions was defined as $\mathrm{p}<0.05$. All tests of significance were two-sided. SAS ${ }^{\circledR}$ version 9.4 (Cary, N.C., USA) was used for all analyses.

Atrial Ectopy and Stroke

\section{Results}

A total of 22,975 (mean age $64 \pm 9.2 ; 56 \%$ women; $40 \%$ black) participants were included in the final analytical sample. There were 1,687 (7.3\%) participants with evidence of PACs on the baseline electrocardiogram. Baseline characteristics stratified by the presence of PACs are shown in table 1.

A higher incidence rate (per 1,000 person-years) of ischemic stroke was observed among those with PACs (incidence rate $6.0,95 \%$ CI 4.7-7.6) than participants without PACs (incidence rate 3.2, 95\% CI 2.9-3.4; ta- 
Fig. 1. Cumulative incidence of ischemic stroke by the presence of PACs. Cumulative incidence curves are statistically different $(\log$-rank $\mathrm{p}<0.0001)$. A higher incidence rate (per 1,000 person-years) of ischemic stroke was observed among those with PACs (incidence rate 6.0, 95\% CI 4.77.6) than those without PACs (incidence rate $3.2,95 \%$ CI 2.9-3.4).

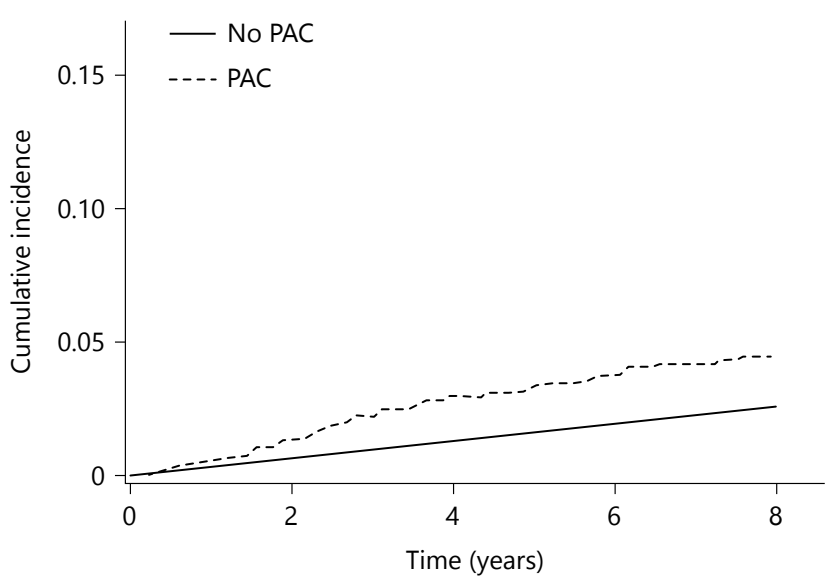

Table 3. Risk of ischemic stroke by age, sex, and race

\begin{tabular}{|c|c|c|c|c|c|}
\hline Subgroup & $\begin{array}{l}\text { Model } 1^{*} \\
\text { HR }(95 \% \text { CI) }\end{array}$ & $\mathrm{p}$ value & $\begin{array}{l}\text { Model } 2^{\dagger}, \\
\text { HR }(95 \% \text { CI })\end{array}$ & $\mathrm{p}$ value & p-interaction ${ }^{\ddagger}$ \\
\hline Age, years & & & & & 0.19 \\
\hline$\geq 65$ & $1.41(1.06-1.88)$ & 0.019 & $1.35(1.01-1.80)$ & 0.041 & \\
\hline Sex & & & & & 0.0095 \\
\hline Female & $1.81(1.29-2.55)$ & 0.0007 & $1.82(1.29-2.56)$ & 0.0007 & \\
\hline Race & & & & & 0.95 \\
\hline Black & $1.43(0.97-2.09)$ & 0.070 & $1.39(0.95-2.04)$ & 0.093 & \\
\hline White & $1.32(0.93-1.87)$ & 0.12 & $1.32(0.93-1.87)$ & 0.12 & \\
\hline
\end{tabular}

* Adjusted for age, sex, race, age* race, education, income and region of residence.

† Adjusted for Model 1 covariates plus systolic blood pressure, smoking, diabetes, low-density lipoprotein cholesterol, left ventricular hypertrophy, antihypertensive medications, lipid-lowering therapies, aspirin, and coronary heart disease.

‡ Interactions tested using Model 2.

ble 2). The cumulative incidence of ischemic stroke by the presence of PACs is depicted in figure 1 and the estimates were statistically different $(\log$ rank $\mathrm{p}<0.0001)$.

In a Cox regression model adjusted for stroke risk factors and potential confounders, PACs were significantly associated with an increased risk of ischemic stroke (table 2). The relationship was limited to non-lacunar infarcts (HR 1.42, 95\% CI 1.08-1.87) and not lacunar strokes (HR 1.01, 95\% CI 0.51-2.03). An interaction by sex was detected, with the association between PACs and ischemic stroke being stronger among women (HR 1.82, 95\% CI 1.29-2.56) than men (HR 1.03, 95\% CI 0.69-1.52; p-interaction $=0.0095$; table 3 ). In a sensitivity analysis in which participants with premature ventricular contrac- tions were included, PACs remained significantly associated with ischemic stroke (HR 1.35, 95\% CI 1.05-1.75) in Model 2. A trend for significance was noted between PACs and recurrent ischemic stroke (HR 1.52, 95\% CI $0.94-2.45 ; \mathrm{p}=0.086)$.

\section{Discussion}

In this analysis from REGARDS, PACs detected on the routine electrocardiogram were associated with an increased risk of ischemic stroke and this association was limited to non-lacunar infarcts. An interaction was detected by sex, with the stroke risk in women nearly dou- 
bling the risk observed in men. Overall, our findings suggest that PACs detected during a screening electrocardiogram recording are not benign entities and provide further support for the hypothesis that atrial ectopy is an independent stroke risk factor.

Several recent reports have examined the association between atrial ectopy and ischemic stroke. A study from the Copenhagen Holter Study has shown that excessive supraventricular ectopy (defined as $\geq 30$ supraventricular ectopic complexes per hour or as any episodes with runs of $\geq 20$ supraventricular ectopic complexes) detected form 48-hour ambulatory electrocardiogram monitoring is associated with an increased ischemic stroke risk (HR 2.37, 95\% CI 1.02-5.50) [6]. A report from the same cohort also has shown that excessive PACs (defined as $\geq 30$ PACs/hour daily or any runs of $\geq 20$ PACs) are associated with an increased risk of ischemic stroke independent of atrial fibrillation (HR 2.00, 95\% CI 1.16-3.45) [5]. Additionally, a retrospective cohort study using administrative claims data from all emergency department encounters and hospitalizations at nonfederal acute care hospitals in California has linked paroxysmal supraventricular tachycardia with incident ischemic stroke during subsequent hospitalizations [13]. In aggregate, these prior reports suggest that atrial rhythm disturbances other than atrial fibrillation, including PACs, are associated with an increased risk of ischemic stroke.

Our findings further support that PACs are associated with an increased risk of ischemic stroke, and suggest that PACs increase the risk for cardiac thromboembolism, as the association was limited to non-lacunar events. More importantly, our results extend prior work to include PACs detected on the routine electrocardiogram, the most commonly used tool for cardiac risk assessment. This finding has important implications regarding stroke risk stratification since long-term ambulatory electrocardiographic monitoring is not feasible in clinical practice. Therefore, the observation that PACs detected on the screening electrocardiogram are not benign findings provides clinicians with another valuable clinical measurement to assess stroke risk. Our data also show that the stroke risk associated with PACs is much higher among women compared with men. Women have been shown to confer a higher risk of stroke in atrial fibrillation and a similar phenomenon possibly exists with PACs [14]. Although our data suggest that atrial ectopy detected on a routine electrocardiogram is associated with an increased stroke risk, further research is needed to determine the causal relationship between both conditions. Also, it is possible that therapies to reduce atrial ectopy and anticoagulation strategies in persons with frequent PACs are beneficial. However, clinical trials are necessary to evaluate the efficacy of such approaches before changes in clinical practice are recommended.

Several mechanisms have been proposed to explain the association between atrial ectopy and ischemic stroke. Possibly, PACs serve as acute triggers for the development of atrial fibrillation and this arrhythmia serves as an intermediate event. This is supported by findings that link PACs with atrial fibrillation development $[4,6]$. However, the stroke risk associated with PACs remains after accounting for documented cases of atrial fibrillation [5]. Persons with PACs also possibly have left atrial disease in which atrial thromboembolism is possible due to reduced left atrial contractility, leading to reductions in left atrial stroke volume and kinetic energy [15-17]. This concept of atrial cardiopathy is supported by recent findings that have associated left atrial disease with ischemic stroke independent of atrial fibrillation $[16,18,19]$. Additionally, it is possible that persons with PACs have poor risk factor profiles that increase their risk of ischemic stroke due to the known association between PACs and several stroke risk factors (e.g., advanced age, dyslipidemia, and physical inactivity) [20]. Although we offer several explanations that link PACs with ischemic stroke events, further research is needed to elucidate the underlying mechanisms that explain our findings.

The current study should be interpreted in the context of certain limitations. Several baseline covariates were selfreported and possibly subjected our analysis to recall bias. PACs were detected at baseline on a single electrocardiogram recording and it is possible that observed relationship changes with long-term measurements (e.g., Holter monitors). However, it is likely that more PACs would have been detected if long-term ambulatory monitoring had been used. We do not know of any reason to suggest that the resulting bias, if any, would have been differential in nature, rather than merely reducing effect estimates toward the null. We classified PACs as present or absent and it is possible that a stronger relationship exists between PACs and stroke by the frequency of PACs. Additionally, we included several covariates in our multivariable models but we acknowledge that other unmeasured factors (e.g., silent ischemia and cardiac structural abnormalities) possibly influenced the association between PACs and stroke.

In conclusion, our findings suggest that PACs detected on the routine electrocardiogram are associated with an increased risk of non-lacunar ischemic strokes, especially in women. Our results challenge the concept that atrial ectopy on the screening electrocardiogram is a benign finding, and further support recent evidence linking 
PACs with ischemic stroke. Future research is needed to determine if persons with atrial ectopy on the resting electrocardiogram with poor risk factor profiles will benefit from anticoagulation strategies to reduce the risk of ischemic stroke.

\section{Acknowledgments}

The authors thank the other investigators, the staff and the participants of the REGARDS study for their valuable contributions. A full list of participating REGARDS investigators and institutions can be found at http://www.regardsstudy.org.

\section{Funding}

This research project is supported by a cooperative agreement U01 NS041588 from the National Institute of Neurological Disorders and Stroke, National Institutes of Health, Department of Health and Human Service. The content is solely the responsibility of the authors and does not necessarily represent the official views of the National Institute of Neurological Disorders and Stroke or the National Institutes of Health.

\section{Disclosure Statement}

The authors declare no conflicts of interest.

\section{References}

1 Christensen H, Boysen G, Christensen AF, Johannesen HH: Insular lesions, ECG abnormalities, and outcome in acute stroke. J Neurol Neurosurg Psychiatry 2005;76:269-271.

2 Perez MV, Dewey FE, Marcus R, Ashley EA, Al-Ahmad AA, Wang PJ, Froelicher VF: Electrocardiographic predictors of atrial fibrillation. Am Heart J 2009;158:622-628.

3 Chong BH, Pong V, Lam KF, Liu S, Zuo ML, Lau YF, Lau CP, Tse HF, Siu CW: Frequent premature atrial complexes predict new occurrence of atrial fibrillation and adverse cardiovascular events. Europace 2012;14:942947.

4 Dewland TA, Vittinghoff E, Mandyam MC, Heckbert SR, Siscovick DS, Stein PK, Psaty BM, Sotoodehnia N, Gottdiener JS, Marcus GM: Atrial ectopy as a predictor of incident atrial fibrillation: a cohort study. Ann Intern Med 2013;159:721-728.

5 Larsen BS, Kumarathurai P, Falkenberg J, Nielsen OW, Sajadieh A: Excessive atrial ectopy and short atrial runs increase the risk of stroke beyond incident atrial fibrillation. J Am Coll Cardiol 2015;66:232-241.

6 Binici Z, Intzilakis T, Nielsen OW, Køber L, Sajadieh A: Excessive supraventricular ectopic activity and increased risk of atrial fibrillation and stroke. Circulation 2010;121:19041911.

7 Howard VJ, Cushman M, Pulley L, Gomez CR, Go RC, Prineas RJ, Graham A, Moy CS, Howard G: The reasons for geographic and racial differences in stroke study: objectives and design. Neuroepidemiology 2005;25: 135-143.
8 Agarwal SK, Chao J, Peace F, Judd SE, Kissela B, Kleindorfer D, Howard VJ, Howard G, Soliman EZ: Premature ventricular complexes on screening electrocardiogram and risk of ischemic stroke. Stroke 2015;46:13651367.

9 Prineas RJ, Crow RS, Zhang ZM: The Minnesota Code Manual of Electrocardiographic Findings: Standards and Procedures for $\mathrm{Mea}-$ surement and Classification, ed 2. London, Springer, 2010.

10 Howard VJ, Kleindorfer DO, Judd SE, McClure LA, Safford MM, Rhodes JD, Cushman M, Moy CS, Soliman EZ, Kissela BM, Howard G: Disparities in stroke incidence contributing to disparities in stroke mortality. Ann Neurol 2011;69:619-627.

11 Stroke - 1989. Recommendations on stroke prevention, diagnosis, and therapy. Report of the WHO task force on stroke and other cerebrovascular disorders. Stroke 1989;20:14071431.

12 Ringelstein EB, Koschorke S, Holling A, Thron A, Lambertz H, Minale C: Computed tomographic patterns of proven embolic brain infarctions. Ann Neurol 1989;26:759765.

13 Kamel H, Elkind MS, Bhave PD, Navi BB, Okin PM, Iadecola C, Devereux RB, Fink ME: Paroxysmal supraventricular tachycardia and the risk of ischemic stroke. Stroke 2013;44: 1550-1554.

14 Lip GY, Nieuwlaat R, Pisters R, Lane DA, Crijns HJ: Refining clinical risk stratification for predicting stroke and thromboembolism in atrial fibrillation using a novel risk factor- based approach: the euro heart survey on atrial fibrillation. Chest 2010;137:263-272.

15 Goyal SB, Spodick DH: Electromechanical dysfunction of the left atrium associated with interatrial block. Am Heart J 2001;142:823827.

16 Kamel H, O’Neal WT, Okin PM, Loehr LR, Alonso A, Soliman EZ: Electrocardiographic left atrial abnormality and stroke subtype in the atherosclerosis risk in communities study. Ann Neurol 2015;78:670-678.

17 O'Neal WT, Zhang ZM, Loehr LR, Chen LY, Alonso A, Soliman EZ: Electrocardiographic advanced interatrial block and atrial fibrillation risk in the general population. Am J Cardiol 2016;117:1755-1759.

18 Kamel H, Bartz TM, Longstreth WT Jr, Okin PM, Thacker EL, Patton KK, Stein PK, Gottesman RF, Heckbert SR, Kronmal RA, Elkind MS, Soliman EZ: Association between left atrial abnormality on ECG and vascular brain injury on MRI in the cardiovascular health study. Stroke 2015;46:711-716.

19 O'Neal WT, Kamel H, Zhang ZM, Chen LY, Alonso A, Soliman EZ: Advanced interatrial block and ischemic stroke: the atherosclerosis risk in communities study. Neurology 2016; 87:352-356.

20 Conen D, Adam M, Roche F, Barthelemy JC, Felber Dietrich D, Imboden M, Kunzli N, von Eckardstein A, Regenass S, Hornemann T, Rochat T, Gaspoz JM, Probst-Hensch N, Carballo D: Premature atrial contractions in the general population: frequency and risk factors. Circulation 2012;126:23022308 . 